Research Paper

\title{
Circulating microparticles are prognostic biomarkers in advanced non-small cell lung cancer patients
}

\author{
Chin-Chou Wang ${ }^{1,2,3, *}$, Chia-Cheng Tseng ${ }^{1,4, *}$, Huang-Chih Chang ${ }^{1,4}$, Kuo-Tung \\ Huang ${ }^{1,4}$, Wen-Feng Fang ${ }^{1,3}$, Yu-Mu Chen ${ }^{1}$, Cheng-Ta Yang ${ }^{5}$, Chang-Chun Hsiao ${ }^{4,6, * *}$, \\ Meng-Chih Lin ${ }^{1, * *}$, Chi-Kung Ho ${ }^{2, * *}$ and Hon-Kan Yip ${ }^{6,7,8,9,10, * *}$ \\ ${ }^{1}$ Division of Pulmonary and Critical Care Medicine, Department of Internal Medicine, Kaohsiung Chang Gung Memorial \\ Hospital, Chang Gung University College of Medicine, Kaohsiung, Taiwan \\ ${ }^{2}$ Department of Public Health, Kaohsiung Medical University, Kaohsiung, Taiwan \\ ${ }^{3}$ Department of Respiratory Care, Chang Gung University of Science and Technology, Chiayi Campus, Chiayi, Taiwan \\ ${ }^{4}$ Graduate Institute of Clinical Medical Sciences, Chang Gung University College of Medicine, Kaohsiung, Taiwan \\ ${ }^{5}$ Department of Pulmonary and Critical Care Medicine, Chang Gung Memorial Hospital, Chang Gung University College of \\ Medicine, Taoyuan, Taiwan \\ ${ }^{6}$ Center for Shockwave Medicine and Tissue Engineering, Kaohsiung Chang Gung Memorial Hospital and Chang Gung \\ University College of Medicine, Kaohsiung, Taiwan \\ ${ }^{7}$ Division of cardiology, Department of Internal Medicine, Kaohsiung Chang Gung Memorial Hospital and Chang Gung \\ University College of Medicine, Kaohsiung, Taiwan \\ ${ }^{8}$ Department of Medical Research, China Medical University Hospital, China Medical University, Taichung, Taiwan \\ ${ }^{9}$ Department of Nursing, Asia University, Taichung, Taiwan \\ ${ }^{10}$ Institute for Translational Research in Biomedicine, Kaohsiung Chang Gung Memorial Hospital, Kaohsiung, Taiwan \\ *These authors contributed equally to this work and should be considered co-first authors \\ ** These authors contributed equally to this work and should be considered co-correspondence authors \\ Correspondence to: Hon-Kan Yip, email: han.gung@msa.hinet.net \\ Keywords: advanced non-small cell lung cancer, microparticles, disease control, disease progression \\ Received: January 09, $2017 \quad$ Accepted: April 25, $2017 \quad$ Published: June 06, 2017 \\ Copyright: Wang et al. This is an open-access article distributed under the terms of the Creative Commons Attribution License 3.0 \\ (CC BY 3.0), which permits unrestricted use, distribution, and reproduction in any medium, provided the original author and source \\ are credited.
}

\section{ABSTRACT}

We investigated whether circulating microparticles (MPs) could serve as prognostic biomarkers in non-small cell lung cancer (NSCLC) patients. We enrolled 25 control subjects and 136 NSCLC patients categorized into disease-progression ( $D P, n=42$ ) and disease-control ( $D C, n=94$ ) groups. Flow cytometric analysis showed that levels of four types of circulating microparticles (EDAC-MPs, EDAp-MPs, PDAC-MPs and PDAp-MPs) were higher in the study patients than the control subjects $(P<0.04)$. DP patients showed poor initially performance status and more non-adenocarcinomas than DC patients. DC patients showed more EGFR mutations and poorer performance to targeted therapy than DP patients $(P<\mathbf{0 . 0 1})$. Three months after therapy, the levels of all four types of circulating MPs were lower in DC than DP patients $(P<0.02)$, and were comparable to the levels in control subjects. In addition, the levels of circulating MPs after 3 months accurately predicted one-year prognostic outcomes $(P<0.05)$. This study showed that circulating MPs are valuable prognostic biomarkers in advanced NSCLC patients.

\section{INTRODUCTION}

Advanced lung cancer (LC) is a leading cause of cancer deaths worldwide [1-4]. Nearly $95 \%$ of all lung cancers are either small cell lung cancer (SCLC) or non- small cell lung cancer (NSCLC). Current treatments include a combination of traditional surgical interventions and adjunctive radiation and chemotherapy. Molecularly targeted drugs for LC include epidermal growth factor receptor tyrosine kinase inhibitors (EGFR TKI) like 
gefitinib, erlotinib, and afatinib [5-9], and anaplastic lymphoma kinase tyrosine kinase inhibitors (ALK TKI) like crizotinib [10]. However, the overall long-term survival rate from lung cancer is extremely low [11-14]. Despite advanced technology, nearly $50 \%$ of lung cancer patients are diagnosed at an advanced stage [4]. Thus, better understanding of the lung cancer pathogenesis and development of effective molecular and cellular biomarkers [4] are necessary to detect cancer early and improve therapeutic outcomes [15-17]. The development of serum biomarkers like microparticles would be useful to predict prognostic outcomes in LC [18-21].

Microparticles (MPs) or membrane-bound vesicles are small fragments of the plasma membrane released by activated and/or apoptotic cells. The MPs ranging from 0.1 to $1.0 \mu \mathrm{m}$ in size circulate in blood and other body fluids and are known to mediate inflammation and thrombosis [22-31]. Additionally, MPs have differential effects on angiogenesis depending on their origin $[22,26$, 28-31]. Microparticles from platelets promote capillary network formation and production of pro-angiogenic factors $[22,24,28,30,32]$. In contrast, both endothelialand lymphocyte-derived MPs possess either pro- or antiangiogenic properties depending on the stimuli $[28,29]$.

Circulating MPs are also associated with a wide range of diseases including LC [28, 32-39]. Circulating endothelial-derived activated MPs (EDAc-MPs) were useful in predicting 1-year morality in advanced stage NSCLC patients [20]. However, since majority of the patients had received palliative treatment prior to enrolment in our previous study, the PDAp-MPs (platelet-derived apoptotic MPs), PDAc-MPs (platelet-derived activated MPs), and EDAp-MPs (endothelial-derived apoptotic MPs) were not prognostic [20]. Therefore, we conducted this prospective study by measuring the circulating levels of MPs in advanced stage NSCLC patients to analyze their prognostic outcomes in advanced stage NSCLC patients.

\section{RESULTS}

\section{Baseline circulating levels of four types microparticles in study subjects}

The circulating levels of the PDAc-MPs, PDApMPs, EDAc-MPs and EDAp-MPs were significantly higher in advanced NSCLC patients compared to control subjects. This suggested that the circulating MPs are useful diagnostic biomarkers for advanced NSCLC patients (Table 1 and Figure 1).

\section{Baseline characteristics of study patients}

The disease control (DC) and disease progression (DP) groups had similar parameters like gender, weight, height, surface area, mass index and incidence of smoking status. The serum levels of total cholesterol, sugar, glutamic oxaloacetic transaminase (GOT) and glutamic pyruvic transaminase (GPT) were also similar between these two groups. Furthermore, the red blood cell (RBC), white blood cell (WBC) and platelet counts as well as circulating levels of carcinoembryonic antigen (CEA) were also similar between the 2 groups. Furthermore, comorbidities like hypertension, diabetes mellitus, coronary artery disease and chronic obstructive lung disease were comparable between DC and DP groups (Table 2).

\section{Lung cancer associated parameters in the study patients}

The cell types of lung cancer (adenocarcinoma or non-adenocarcinoma) were similar between the DC and the DP groups. However, higher epidermal growth factor receptor (EGFR) mutation levels were observed in the DC patients compared to DP group. The incidence of metastasis and the metastatic sites were similar for the two groups of the patients. Also, the two groups showed no differences in stages IIIB or IV. However, the DP patients' performance status was poorer than the DC group, and the DC group of patients was easily met with target therapy (Table 3 ).

\section{Circulating microparticle levels in DC and DP patients}

Table 4 shows the changes in circulating levels of PDAc-MPs, PDAp-MPs, EDAc-MPs, EDAp-MPs between the DC and DP patients prior to and at the end of $1^{\text {st }}$ and $3^{\text {rd }}$ months after pharmacological intervention. The circulating levels of the four types of MPs were similar between the DC and DP groups prior to and at the end of the first month after pharmacological therapy (Figure 2). However, at the end of the third month after pharmacological therapy, all the four types of MPs were significantly lower in the DC group than in the DP group (Figure 2). This was corroborated by positive $\triangle$ EDAp-MP, $\triangle$ EDAc-MP, $\triangle \mathrm{PDAp}-\mathrm{MP}$ and $\triangle \mathrm{PDAc}$ MP values associated with DP. These findings demonstrated that the pharmacological therapy lowered the circulating MPs in the DC group (Table 4 and Figure 2).

\section{Comparing effects of chemotherapy and targeted therapy on circulating microparticle levels}

Table 5 compares changes in circulating levels of PDAc-MPs, PDAp-MPs, EDAc-MPs, EDAp-MPs in the patients that received chemotherapy or targeted therapy. The lung cancer patients with EGFR mutant type were treated with EGFR TKI agents and those with EGFR wildtype subgroup were treated with chemotherapy based on guideline recommendations. The flow cytometric analysis showed that the circulating levels of the four types of MPs were similar between the chemotherapy and targeted therapy patients prior to and at the end of the first month after pharmacological therapy. However, at the end of the third month after pharmacological therapy, all the four types of MPs were significantly lower in patients that 
Table 1: Comparison of circulating levels of four types microparticles between lung cancer patients and healthy control group

\begin{tabular}{lccc}
\hline Variables & Study Group $(\mathbf{n}=\mathbf{1 3 6})^{*}$ & Control Group $(\mathbf{n}=\mathbf{2 5})$ & P-value \\
\hline PDAc-MPs & $184866.40 \pm 723526.40$ & $20334.75 \pm 25884.62$ & 0.009 \\
PDAp-MPs & $30988.10 \pm 95402.90$ & $13725.08 \pm 5401.12$ & 0.038 \\
EDAc-MPs & $7196.16 \pm 33141.41$ & $598.54 \pm 582.43$ & 0.022 \\
EDAp-MPs & $27171.17 \pm 105285.34$ & $5486.17 \pm 4331.44$ & 0.018 \\
\hline
\end{tabular}

Data are expressed as mean $\pm \mathrm{SD}$.

PDAc-MPs = platelet-derived activated microparticles; PDAp-MPs = platelet-derived apoptotic MPs; EDAc-MPs = endothelial-derived activated MPs; EDAp-MPs = endothelial-derived apoptotic MPs.

* indicated the blood sample was drawn prior to treatment.
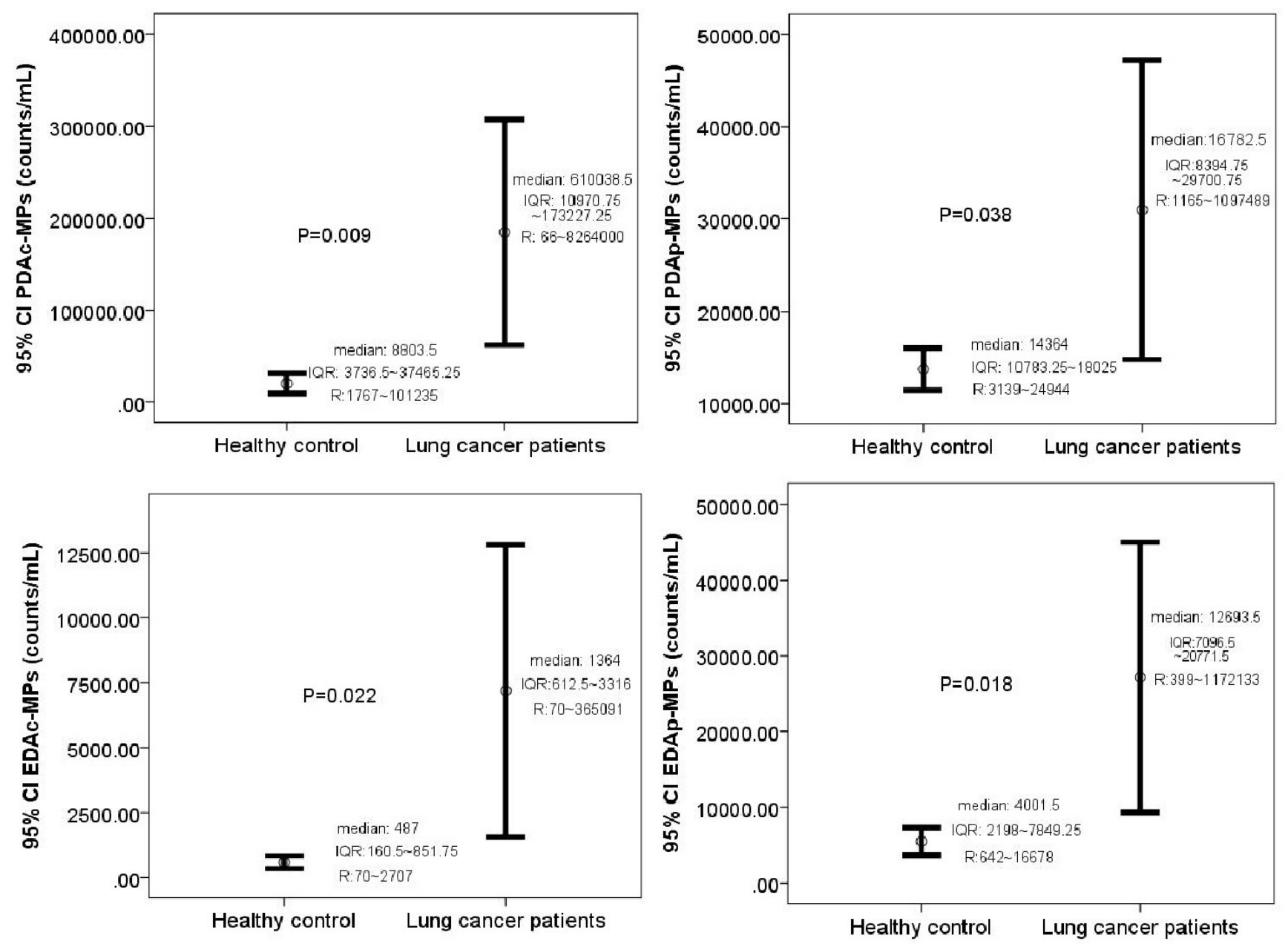

Figure 1: Comparison of baseline levels of circulating microparticles in the study and control subjects. Circulating levels of (A) Platelet-derived activated MPs (PDAc-MPs; $\mathrm{p}=0.009$ ), (B) Platelet-derived apoptotic MPs (PDAp-MPs; p = 0.038), (C) Endothelial-derived activated MPs (EDAc-MPs; $\mathrm{p}=0.022$ ) and (D) Endothelial-derived apoptotic MPs (EDAp-MPs; $\mathrm{p}=0.018$ ) in study and control subjects. Note: $\mathrm{CI}=$ confidence intervals. 
Table 2: Baseline Characteristics of 136 Study Patients

\begin{tabular}{|c|c|c|c|}
\hline Variable & Disease Progression $(n=42)$ & Disease Control $(n=94)$ & P-value \\
\hline Age & $62.69 \pm 10.80$ & $65.01 \pm 10.23$ & 0.244 \\
\hline Sex (male) & $64.3 \%(27)$ & $61.7 \%(58)$ & 0.849 \\
\hline Body weight (kg) & $65.47 \pm 19.72$ & $73.04 \pm 35.09$ & 0.112 \\
\hline Body height $(\mathrm{cm})$ & $162.45 \pm 23.02$ & $147.34 \pm 36.17$ & 0.004 \\
\hline Body surface area $\left(\mathrm{m}^{2}\right)$ & $1.67 \pm 0.18$ & $1.64 \pm 0.18$ & 0.338 \\
\hline Body mass index $\left(\mathrm{kg} / \mathrm{m}^{2}\right)$ & $23.61 \pm 3.27$ & $23.31 \pm 3.66$ & 0.642 \\
\hline Smoking status & $50 \%(21)$ & $54.2 \%(51)$ & 0.711 \\
\hline Total cholesterol (mg/dL) & $176.33 \pm 49.09$ & $182.25 \pm 45.51$ & 0.506 \\
\hline Triglyceride (mg/dL) & $178.75 \pm 81.92$ & $193.12 \pm 86.47$ & 0.365 \\
\hline Ac sugar $(\mathrm{mg} / \mathrm{dL})$ & $123.17 \pm 48.15$ & $128.36 \pm 66.16$ & 0.608 \\
\hline Creatinine & $0.90 \pm 0.34$ & $0.89 \pm 0.35$ & 0.928 \\
\hline $\mathrm{Na}$ & $136.69 \pm 8.20$ & $137.10 \pm 6.91$ & 0.766 \\
\hline $\mathrm{K}$ & $3.99 \pm 0.59$ & $3.85 \pm 0.57$ & 0.224 \\
\hline $\begin{array}{l}\text { Aspartate aminotransferase } \\
\text { (IU) }\end{array}$ & $26.38 \pm 16.36$ & $25.62 \pm 12.22$ & 0.766 \\
\hline $\begin{array}{l}\text { Alanine aminotransferase } \\
\text { (IU) }\end{array}$ & $31.14 \pm 38.10$ & $28.79 \pm 27.36$ & 0.685 \\
\hline $\begin{array}{l}\text { White blood cell count }\left(\times 10^{3} /\right. \\
\mathrm{mL})\end{array}$ & $8.23 \pm 5.20$ & $7.92 \pm 3.48$ & 0.690 \\
\hline $\begin{array}{l}\text { Red blood cell count }\left(\times 10^{6} /\right. \\
\mathrm{mL})\end{array}$ & $4.41 \pm 0.58$ & $4.44 \pm 0.73$ & 0.814 \\
\hline Platelet count $\left(\times 10^{3} / \mathrm{mL}\right)$ & $27.7 \pm 12.7$ & $26.4 \pm 10.5$ & 0.539 \\
\hline CEA & $357.16 \pm 1760.44$ & $75.88 \pm 166.13$ & 0.302 \\
\hline \multicolumn{4}{|l|}{ Underlying comorbidity } \\
\hline Hypertension & $47.6 \%(20)$ & $39.4 \%(37)$ & 0.452 \\
\hline Diabete mellitus & $19 \%(8)$ & $18.1 \%(17)$ & 1.000 \\
\hline COPD & $9.5 \%(4)$ & $12.1 \%(11)$ & 1.000 \\
\hline CAD & $14.3 \%(6)$ & $22.3 \%(21)$ & 0.355 \\
\hline
\end{tabular}

Data are expressed as mean $\pm \mathrm{SD}$ or $\%$ (n).

received targeted therapy compared to those that received chemotherapy. (Table 5)

\section{Circulating levels of microparticles in one-year survivors and non-survivors}

We observed that the four types of circulating MPs did not differ between 1-year survivors and nonsurvivors prior to and at one month time intervals after pharmacological intervention. However, at the end of the third month after pharmacological intervention, except for EDAc-MPs, the other three types of MPs were significantly higher in the one-year non-survivors than in one-year survivors. Additionally, PDAc-MPs and EDAc-MPs levels were significantly higher in 1-year non-survivors than in 1 -year survivors. These findings suggest that the circulating MPs can serve as 1-year prognostic predictors in advanced stage NSCLC patients. (Table 6 and Figure 3)

\section{Receiver operating characteristic (ROC) plot of circulating levels of MPs and CEA in NSCLC patients with progressive and non-progressive disease}

Next, we examined if the circulating levels of the four types of MPs and CEA could predict progressive 
Table 3: Lung Cancer Associated Parameters in 136 Study Patients

\begin{tabular}{|c|c|c|c|}
\hline Variables & Disease Progression $(n=42)$ & Disease Control $(n=94)$ & P-value \\
\hline Cell type & & & 0.049 \\
\hline Adenocarcinoma & $73.8 \%(31)$ & $87.2 \%(82)$ & \\
\hline Non-adenocarcinoma & $26.2 \%(11)$ & $12.8 \%(12)$ & \\
\hline Metastasis & & & 0.355 \\
\hline M0 & $23.8 \%(10)$ & $21.3 \%(20)$ & \\
\hline M1a & $23.8 \%(10)$ & $36.2 \%(34)$ & \\
\hline M1b & $52.4 \%(22)$ & $42.5 \%(40)$ & \\
\hline Stage & & & 0.824 \\
\hline $\mathrm{IIIb}$ & $23.8 \%(10)$ & $21.3 \%(20)$ & \\
\hline IV & $76.2 \%(32)$ & $78.7 \%(74)$ & \\
\hline \multicolumn{4}{|l|}{ Metastatic site } \\
\hline Pleura & $31 \%(13)$ & $41.5 \%(39)$ & 0.259 \\
\hline Lung & $40.5 \%(17)$ & $26.6 \%(25)$ & 0.440 \\
\hline Bone & $28.6 \%(12)$ & $34 \%(32)$ & 0.559 \\
\hline Liver & $7.1 \%(3)$ & $8.5 \%(8)$ & 1.000 \\
\hline Adrenal gland & $7.1 \%(3)$ & $1.1 \%(1)$ & 0.087 \\
\hline Brain & $16.7 \%(7)$ & $12.8 \%(12)$ & 0.596 \\
\hline Performance status & & & 0.008 \\
\hline 0 & $14.3 \%(6)$ & $9.6 \%(9)$ & \\
\hline 1 & $57.1 \%(24)$ & $80.9 \%(76)$ & \\
\hline 2 & $28.6 \%(12)$ & $9.6 \%(9)$ & \\
\hline Therapeutic Intervention & & & 0.000 \\
\hline Target therapy & $11.9 \%(5)$ & $70.2 \%(66)$ & \\
\hline Chemotherapy & $88.1 \%(37)$ & $29.8 \%(28)$ & \\
\hline EGFR status & $11.9 \%(5)$ & $70.2 \%(66)$ & 0.000 \\
\hline
\end{tabular}

Data are expressed as \% (n).

disease in NSCLC patients. As shown in Figure 4 and Table 7, ROC curve analysis showed that the circulating levels of EDAp-MPs, EDAc-MPs, PDApMPs and PDAc-MPs were greater than 10468.5 counts/ $\mathrm{ml}, 3557$ counts $/ \mathrm{ml}, 15055$ counts $/ \mathrm{ml}$ and 62700.5 counts $/ \mathrm{ml}$, respectively at the $3^{\text {rd }}$ month after therapy. These data showed that the 4 types of MPs had high sensitivity and specificity and were good prognostic predictors for advanced stage NSCLC. Further, multivariate logistic regression analysis showed that the EGFR mutant, levels of EDAc-MPs at $3^{\text {rd }}$ month after therapy and the $\triangle$ PDAp-MPs and $\triangle$ PDAc-MPs were independent prognostic predictors in NSCLC patients (Table 8).

\section{DISCUSSION}

In this study, we determined if circulating levels of MPs could predict clinical outcomes in advanced stage NSCLC patients. We observed that circulating levels of the four types of MPs that we analyzed were higher in advanced stage NSCLC patients. The levels of EDAc-MPs, EDAp-MPs, PDAc-MPs, PDAp-MPs did not differ between DC and DP prior to therapeutic intervention. However, by the end of the third month, all the four biomarkers were significantly lower in the DC group compared with the DP group and similar to control subjects. Additionally, the positive net changes of four types of microparticles (i.e., $\triangle \mathrm{EDAp}-\mathrm{MPs}, \triangle \mathrm{EDAc}-\mathrm{MPs}$, 
Table 4: Serial changes of circulating microparticles between disease progression and disease control patients

\begin{tabular}{|c|c|c|c|}
\hline Variables & Disease Progression $(n=42)$ & Disease Control $(n=94)$ & P-value \\
\hline PDAc-MPs (time 1)* & & & 0.554 \\
\hline mean \pm SD & $129713.69 \pm 181619.98$ & $209509.10 \pm 862192.46$ & \\
\hline median & 19720.5 & 11749.5 & \\
\hline interquartile range & $6978.75 \sim 29012.25$ & $7050.25 \sim 18300$ & \\
\hline range & $3307 \sim 408654$ & $399 \sim 1172133$ & \\
\hline PDAp-MPs (time 1)* & & & 0.963 \\
\hline mean \pm SD & $30424.81 \pm 36668.18$ & $31239.79 \pm 112335.20$ & \\
\hline median & 1290.0 & 1432 & \\
\hline interquartile range & $672.25 \sim 3950$ & $603.5 \sim 3220$ & \\
\hline range & $160 \sim 92792$ & $70 \sim 365091$ & \\
\hline EDAc-MPs (time 1)* & & & 0.779 \\
\hline mean \pm SD & $5998.31 \pm 15338.89$ & $7731.37 \pm 38596.88$ & \\
\hline median & 20198.5 & 15393 & \\
\hline interquartile range & $8434.25 \sim 38064$ & $8244.25 \sim 25611$ & \\
\hline range & $1042 \sim 8637.5$ & $1165 \sim 1097489$ & \\
\hline EDAp-MPs (time 1)* & & & 0.816 \\
\hline mean \pm SD & $29695.21 \pm 62524.79$ & $26043.40 \pm 119847.80$ & \\
\hline median & 54283.5 & 26465 & \\
\hline interquartile range & $9166.75 \sim 193062.75$ & $12291.75 \sim 69015.5$ & \\
\hline range & $1042 \sim 863715$ & $66 \sim 8264000$ & \\
\hline PDAc-MPs (time 2$) \dagger$ & & & 0.267 \\
\hline mean \pm SD & $61578.98 \pm 83592.25$ & $94027.07 \pm 106312.33$ & \\
\hline median & 10621 & 11047 & \\
\hline interquartile range & $7148.5 \sim 14540$ & $6145.75 \sim 18297.5$ & \\
\hline range & $1818 \sim 26138$ & $134 \sim 46044$ & \\
\hline PDAp-MPs (time 2) $\dagger$ & & & 0.325 \\
\hline mean \pm SD & $15173.62 \pm 11688.99$ & $18028.77 \pm 17020.22$ & \\
\hline median & 763.5 & 2240.5 & \\
\hline interquartile range & $423.25 \sim 1791.75$ & $765 \sim 4536.75$ & \\
\hline range & $104 \sim 28217$ & $110 \sim 21870$ & \\
\hline EDAc-MPs (time 2) $\dagger$ & & & 0.267 \\
\hline mean \pm SD & $2315.71 \pm 4803.61$ & $3237.34 \pm 3474.93$ & \\
\hline median & 11026 & 12807.5 & \\
\hline interquartile range & $7757.25 \sim 20321$ & $6660.25 \sim 24240.25$ & \\
\hline range & $1239 \sim 53748$ & $122 \sim 10827$ & \\
\hline EDAp-MPs (time 2$) \dagger$ & & & 0.200 \\
\hline mean $\pm \mathrm{SD}$ & $10912.26 \pm 5543.11$ & $12782.32 \pm 8648.35$ & \\
\hline median & 35472 & 61596.5 & \\
\hline interquartile range & $8231.25 \sim 76459.75$ & $24945.5 \sim 124349.5$ & \\
\hline range & 568 425339 & $48 \sim 662081$ & \\
\hline
\end{tabular}




\begin{tabular}{|c|c|c|c|}
\hline Variables & Disease Progression $(n=42)$ & Disease Control $(n=94)$ & P-value \\
\hline PDAc-MPs (time 3$) \ddagger$ & & & 0.009 \\
\hline mean \pm SD & $163110.26 \pm 250042.10$ & $55555.49 \pm 76172.00$ & \\
\hline median & 16907 & 8565.5 & \\
\hline interquartile range & $10666.25 \sim 25465.25$ & $4527.75 \sim 13739.75$ & \\
\hline range & $1050 \sim 66499$ & $723 \sim 45969$ & \\
\hline PDAp-MPs (time 3$) \$$ & & & 0.013 \\
\hline mean \pm SD & $31602.12 \pm 43567.74$ & $13907.85 \pm 13973.76$ & \\
\hline median & 5121 & 1158 & \\
\hline interquartile range & $2212.5 \sim 13313$ & $518 \sim 2915.25$ & \\
\hline range & $471 \sim 127265$ & $99 \sim 8049$ & \\
\hline EDAc-MPs (time 3$) \$$ & & & 0.007 \\
\hline mean $\pm \mathrm{SD}$ & $10551.19 \pm 19882.08$ & $1900.62 \pm 1889.91$ & \\
\hline median & 17211 & 9094 & \\
\hline interquartile range & $8945.75 \sim 32166$ & $5765.75 \sim 16032.75$ & \\
\hline range & $1211 \sim 206185$ & $966 \sim 81818$ & \\
\hline EDAp-MPs (time 3$) \$$ & & & $<0.000$ \\
\hline mean $\pm \mathrm{SD}$ & $19717.12 \pm 13296.76$ & $9966.81 \pm 7436.74$ & \\
\hline median & 110855.5 & 27656.5 & \\
\hline interquartile range & $492545 \sim 221313.75$ & $9829.5 \sim 70369.5$ & \\
\hline range & $4831 \sim 1499326$ & $21 \sim 465579$ & \\
\hline$\triangle$ EDAp-MPs & & & 0.003 \\
\hline Increase & $64.3 \%(27)$ & $36.2 \%(34)$ & \\
\hline Decrease & $35.7 \%(15)$ & $63.8 \%(60)$ & \\
\hline$\triangle$ EDAc-MPs & & & $<0.0001$ \\
\hline Increase & $85.7 \%(36)$ & $44.7 \%(42)$ & \\
\hline Decrease & $14.3 \%(6)$ & $55.6 \%(52)$ & \\
\hline$\triangle \mathrm{PDAp}-\mathrm{MPs}$ & & & 0.036 \\
\hline Increase & $50 \%(21)$ & $30.9 \%(29)$ & \\
\hline Decrease & $50 \%(21)$ & $69.1 \%(65)$ & \\
\hline$\triangle \mathrm{PDAc}-\mathrm{MPs}$ & & & 0.005 \\
\hline Increase & $64.3 \%(27)$ & $37.2 \%(35)$ & \\
\hline Decrease & $35.7 \%(15)$ & $62.8 \%(59)$ & \\
\hline
\end{tabular}

Data expressed as mean $\pm \mathrm{SD}$.

PDAc-MPs = platelet-derived activated microparticles; PDAp-MPs = platelet-derived apoptotic MPs; EDAc-MPs = endothelial-derived activated MPs; EDAp-MPs = endothelial-derived apoptotic MPs.

* indicated the blood sampling was performed prior to any treatment. $\dagger$ indicated the blood sampling was performed at the end of $1^{\text {st }}$ month after pharmacological intervention. $t$ indicated the blood sample was performed at the end of the $3^{\text {rd }}$ month after pharmacological intervention.

$\triangle$ EDAp-MPs: EDApMPs levels that three months after treatment minus initial level $\triangle$ EDAc-MPs: EDAcMPs levels that three months after treatment minus initial level $\triangle$ PDAp-MPs: PDApMPs levels that three months after treatment minus initial level $\triangle$ PDAc-MPs: PDAcMPs levels that three months after treatment minus initial level 
$\triangle$ PDAp-MPs and $\triangle$ PDAc-MPs) between $3^{\text {rd }}$ month and baseline were strongly associated with DP. Furthermore, ROC curve identified that the absolute values at three months and the relative changes of MP values over time (i.e., $\triangle$ EDAp-MPs, $\triangle$ EDAc-MPs, $\triangle$ PDAp-MPs and $\triangle$ PDAc-MPs) had notably higher sensitivity and specificity than that of the CEA level for predicting the prognostic outcomes. Moreover, multivariate logistic regression analysis exhibited that the EGFR mutant, EDAc-MPs level at $3^{\text {rd }}$ month and the net change between baseline and $3^{\text {rd }}$ month (i.e., $\triangle$ PDAp-MPs and $\triangle P D A c-$ MPs) were also independently predictive of DP in NSCLC patients. Accordingly, these suggested that these four types of circulating MPs may be useful biomarkers for predicting prognostic outcomes in LC patients.

This study also showed that the circulating levels EDAc-MPs, EDAp-MPs, PDAc-MPs were higher in
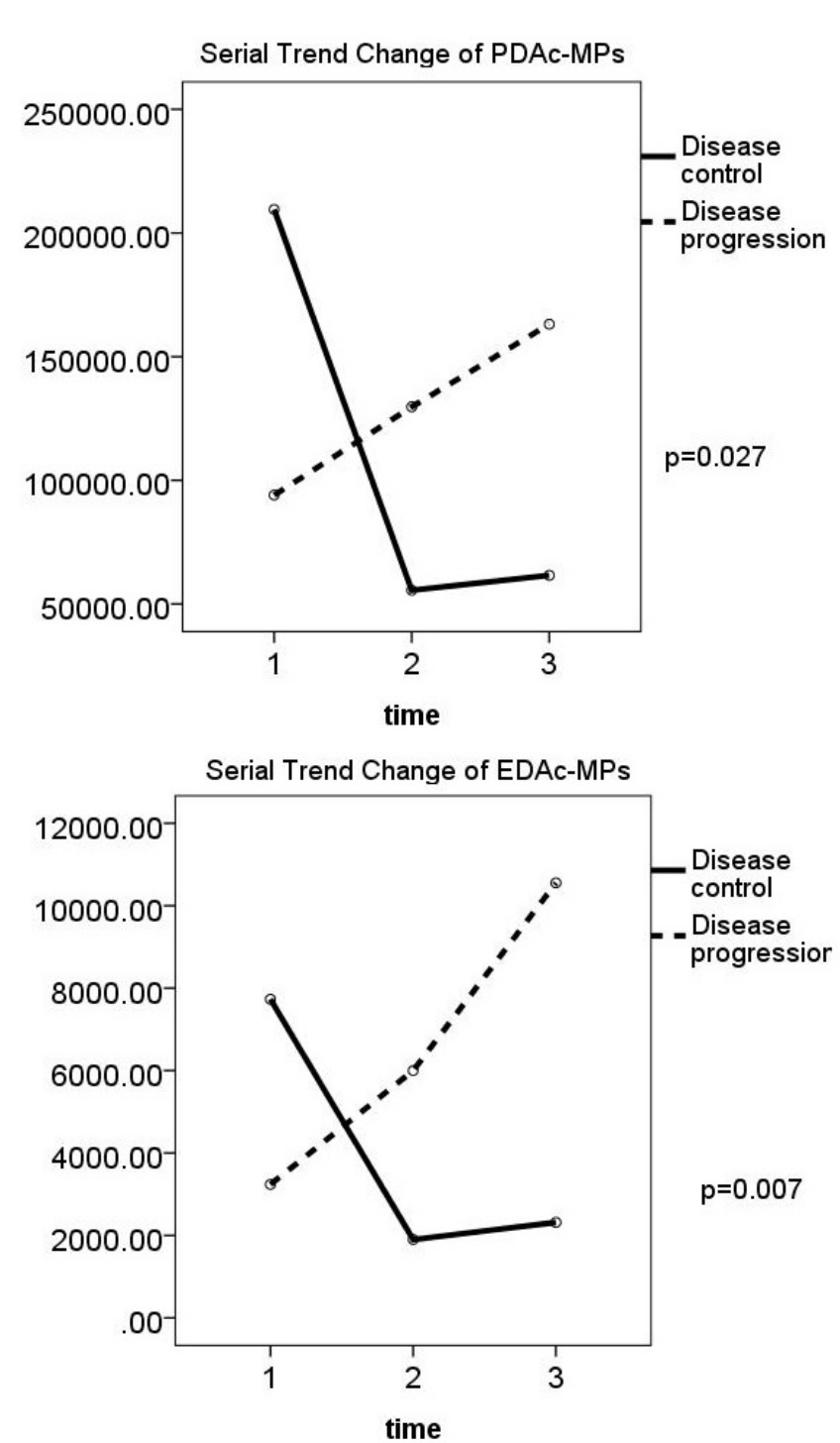

study patients prior to receiving treatment than in control subjects and were consistent with previous studies [1820]. Most importantly, the circulating levels of the four types of MPs in the DC group were significantly lower and comparable to the control subjects at end of the third month in the DC group than in the DP group. Furthermore, the circulating levels of MPs independently predicted the one-year prognostic clinical outcome in the advanced stage NSCLC patients. Thus, our findings highlight that serial measurement of circulating MPs can predict therapeutic response and prognostic outcomes in advanced NSCLC patients, especially when considering the cost and effectiveness of target therapy.

An association between aberrant EGFR mutation activity and better prognostic outcomes has been reported previously [40] [18-20]. Additionally, poor performance status and poorer prognostic outcomes were
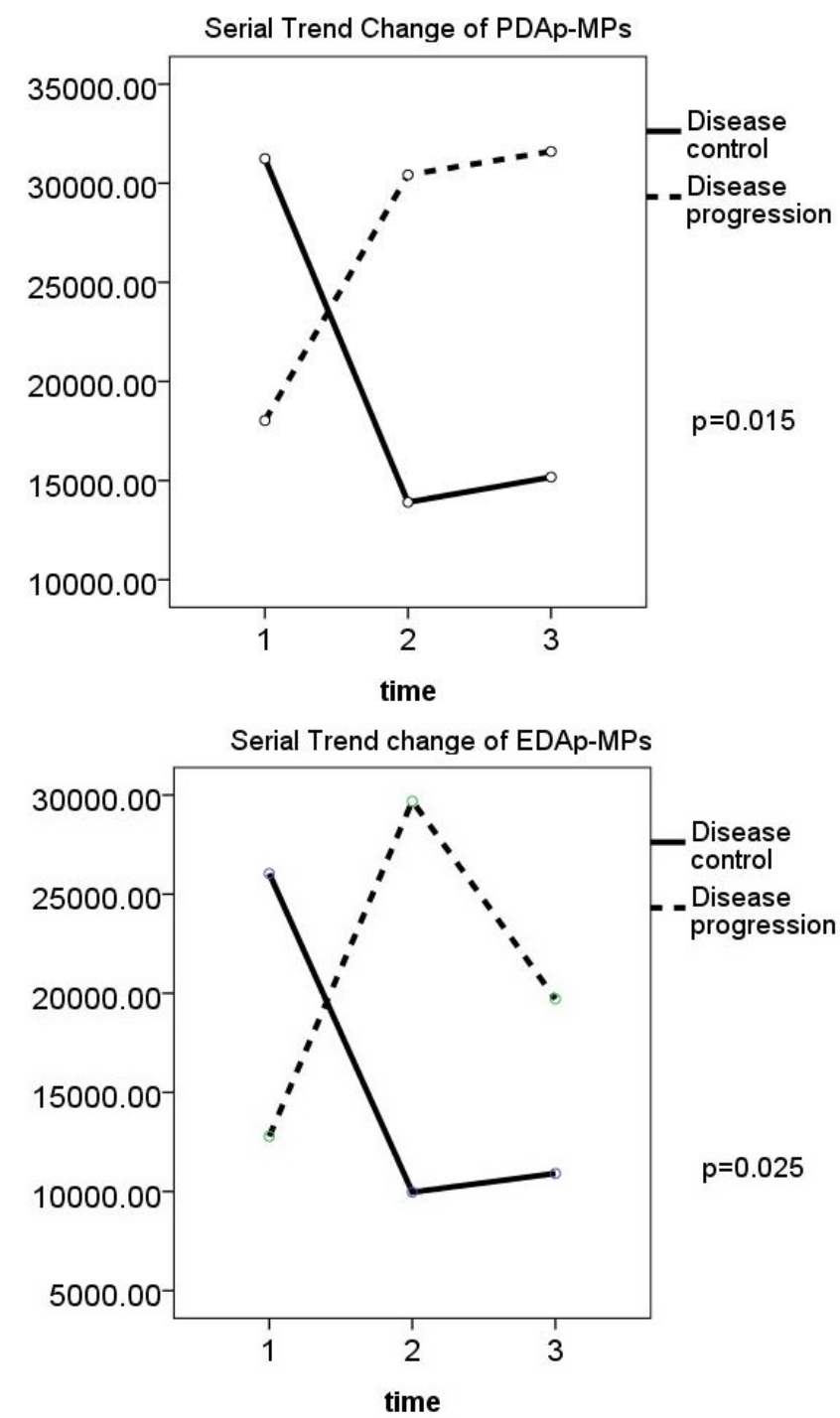

Figure 2: Comparison of changes in levels of the four types of microparticles in disease control (DC) and disease progression (DP) groups. Changes in levels of (A) PDAc-MPs ( $p=0.027)$, (B) PDAp-MPs ( $p=0.015),(\mathbf{C})$ EDAc-MPs ( $p=0.007)$ and (D) EDAp-MPs $(\mathrm{p}=0.025)$ between DC and DP group of patients. 
Table 5: Serial changes of circulating microparticles between chemotherapy and target therapy patients

\begin{tabular}{lccc}
\hline Variables & Chemotherapy $(\mathbf{n = 6 5})$ & Target therapy $(\mathbf{n = 7 1})$ & P-value \\
\hline PDAc-MPs (time 1)* & $223983.58 \pm 1022792.58$ & $149054.89 \pm 224562.98$ & 0.548 \\
PDAp-MPs (time 1)* & $44645.09 \pm 136502.00$ & $18485.23 \pm 13633.03$ & 0.129 \\
EDAc-MPs (time 1)* & $9618.75 \pm 46275.14$ & $9478.30 \pm 12246.30$ & 0.417 \\
EDAp-MPs (time 1)* & $42145.84 \pm 15114.76$ & $13461.90 \pm 10052.02$ & 0.132 \\
PDAc-MPs (time 2) $\dagger$ & $73103.35 \pm 109872.19$ & $93987.94 \pm 91086.41$ & 0.228 \\
PDAp-MPs (time 2) $\dagger$ & $16885.72 \pm 17225.25$ & $17386.25 \pm 14031.42$ & 0.852 \\
EDAc-MPs (time 2) $\dagger$ & $2613.71 \pm 4444.46$ & $3263.08 \pm 3412.56$ & 0.339 \\
EDAp-MPs (time 2) $\dagger$ & $11468.14 \pm 7389.82$ & $12879.21 \pm 8241.49$ & 0.297 \\
PDAc-MPs (time 3) $\dagger$ & $119051.69 \pm 250550.43$ & $61049.11 \pm 83443.71$ & 0.034 \\
PDAp-MPs (time 3) $\dagger$ & $24724.68 \pm 36663.15$ & $14472.15 \pm 14869.75$ & 0.039 \\
EDAc-MPs (time 3) $\dagger$ & $7070.12 \pm 16525.59$ & $2285.21 \pm 2554.22$ & 0.024 \\
EDAp-MPs (time 3) $\dagger$ & $15424.49 \pm 12684.86$ & $10738.13 \pm 7661.49$ & 0.011 \\
\hline
\end{tabular}

Data expressed as mean $\pm \mathrm{SD}$.

PDAc-MPs = platelet-derived activated microparticles; PDAp-MPs = platelet-derived apoptotic MPs; EDAc-MPs = endothelial-derived activated MPs; EDAp-MPs = endothelial-derived apoptotic MPs.

* indicated the blood sampling was performed prior to any treatment. $\dagger$ indicated the blood sampling was performed at the end of $1^{\text {st }}$ month after pharmacological intervention. $\$$ indicated the blood sample was performed at the end of the $3^{\text {rd }}$ month after pharmacological intervention.

Table 6: Serial changes of circulating microparticles between one year survivors and on year non-survivors

\begin{tabular}{lccc}
\hline Variables & $\begin{array}{c}\text { One year non-survivors } \\
(\mathbf{n}=\mathbf{3 4})\end{array}$ & One year survivors $(\mathbf{n}=\mathbf{1 0 2})$ & P-value \\
\hline PDAc-MPs (time 1)* & $106012.59 \pm 119401.35$ & $211151.00 \pm 832025.21$ & 0.465 \\
PDAp-MPs (time 1)* & $22543.09 \pm 19448.73$ & $33803.11 \pm 109590.41$ & 0.553 \\
EDAc-MPs (time 1)* & $5019.29 \pm 7981.37$ & $7921.78 \pm 38015.19$ & 0.660 \\
EDAp-MPs (time 1)* & $29914.29 \pm 68885.70$ & $26256.79 \pm 115164.17$ & 0.862 \\
PDAc-MPs (time 2) $\dagger$ & $86471.00 \pm 96982.29$ & $83184.78 \pm 102325.18$ & 0.334 \\
PDAp-MPs (time 2) $\dagger$ & $14217.09 \pm 9419.38$ & $18123.68 \pm 17079.45$ & 0.486 \\
EDAc-MPs (time 2) $\dagger$ & $3487.41 \pm 5622.06$ & $2774.49 \pm 3205.34$ & 0.098 \\
EDAp-MPs (time 2) $\dagger$ & $11096.35 \pm 5378.55$ & $12574.28 \pm 8505.54$ & 0.870 \\
PDAc-MPs (time 3) $\dagger$ & $188877.00 \pm 267863.35$ & $55402.25 \pm 78140.98$ & 0.009 \\
PDAp-MPs (time 3) $\dagger$ & $23141.74 \pm 38386.77$ & $18115.76 \pm 23505.10$ & 0.007 \\
EDAc-MPs (time 3) $\dagger$ & $12522.74 \pm 21674.66$ & $1921.91 \pm 1842.74$ & 0.365 \\
EDAp-MPs (time 3) + & $17790.21 \pm 12573.57$ & $11373.84 \pm 9379.33$ & 0.007 \\
\hline
\end{tabular}

Data expressed as mean \pm SD.

PDAc-MPs = platelet-derived activated microparticles; PDAp-MPs = platelet-derived apoptotic MPs; EDAc-MPs = endothelial-derived activated MPs; EDAp-MPs = endothelial-derived apoptotic MPs.

* indicated the blood sampling was performed prior to any treatment. $\dagger$ indicated the blood sampling was performed at the end of $1^{\text {st }}$ month after pharmacological intervention. $\$$ indicated the blood sample was performed at the end of the $3^{\text {rd }}$ month after pharmacological intervention. 

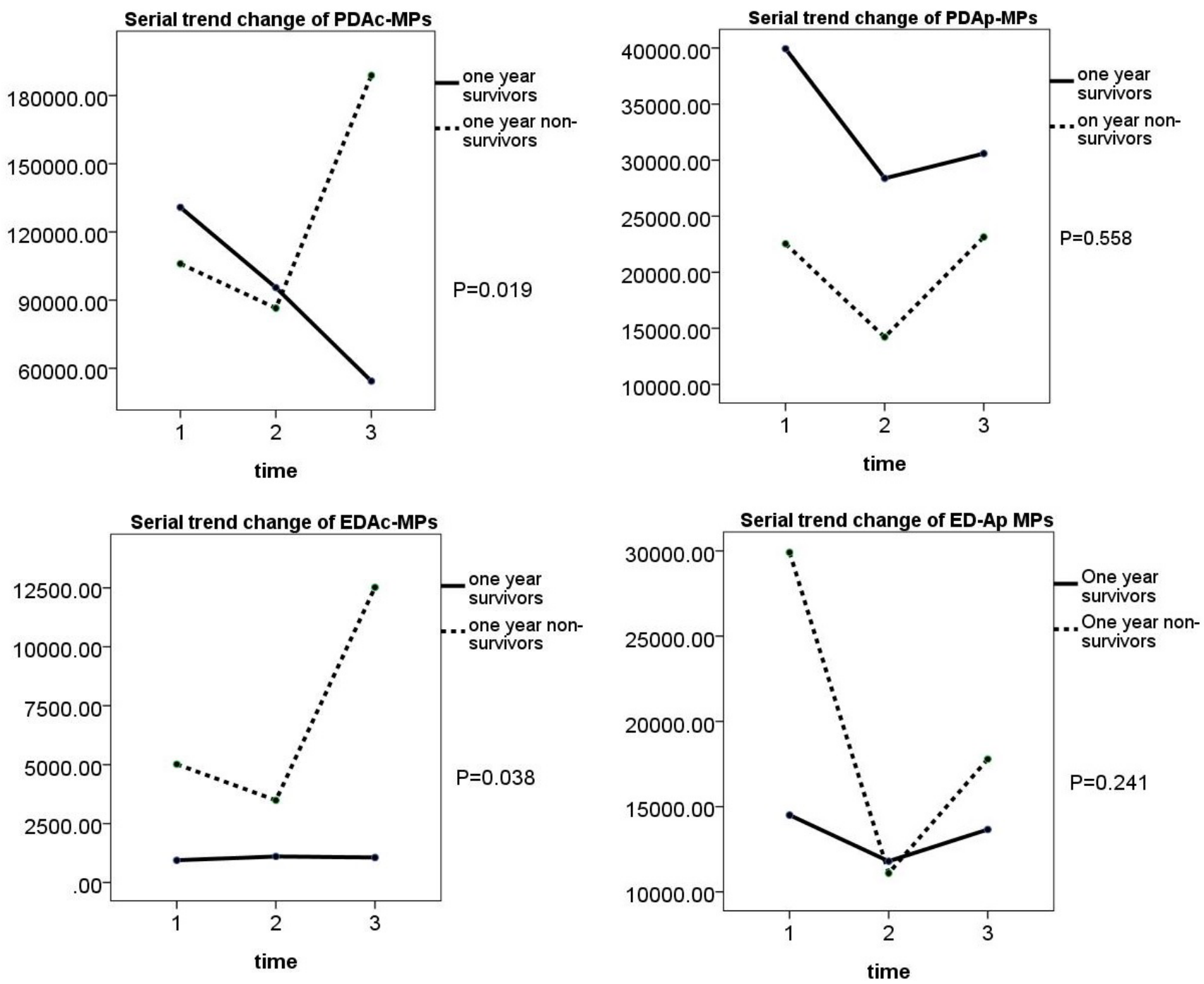

Figure 3: Comparison of changes in levels of the four types of microparticles in one-year survivors and non-survivors. Changes in levels of (A) PDAc-MPs ( $\mathrm{p}=0.019)$, (B) PDAp-MPs ( $\mathrm{p}=0.558),(\mathbf{C})$ EDAc-MPs ( $\mathrm{p}=0.038),(\mathbf{D})$ EDAp-MPs ( $\mathrm{p}=0.241$ in oneyear survivor and non-survivor groups.

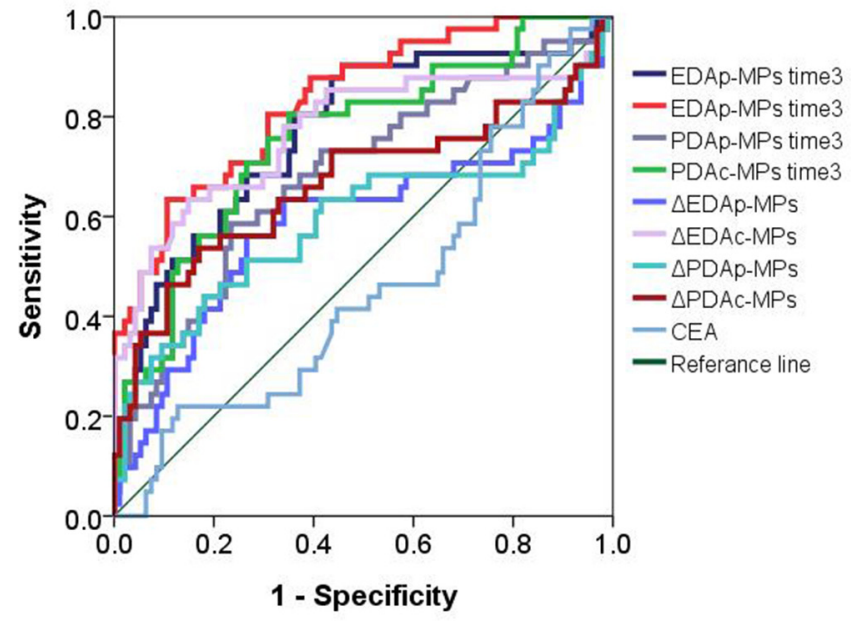

Figure 4: Receiver operating characteristic (ROC) plot showing circulating levels of microparticles (MPs) in progressive and non-progressive NSCLC. 
Table 7: Value of four types of microparticles for predicting progressive disease of NSCLC patients

\begin{tabular}{lccc}
\hline Variables & AUC & P value & 95\% CI \\
\hline EDAp-MPs (time 3) & 0.805 & $<0.000$ & $0.680 \sim 0.861$ \\
EDAc-MPs (time 3) & 0.659 & $<0.000$ & $0.760 \sim 0.907$ \\
PDAp-MPs (time 3) & 0.610 & 0.001 & $0.584 \sim 0.787$ \\
PDAc-MPs (time 3) & 0.707 & $<0.000$ & $0.667 \sim 0.847$ \\
$\Delta$ EDAp-MPs & 0.590 & 0.098 & $0.471 \sim 0.708$ \\
$\Delta$ EDAc-MPs & 0.771 & $<0.000$ & $0.670 \sim 0.872$ \\
$\Delta$ PDAp-MPs & 0.594 & 0.083 & $0.474 \sim 0.714$ \\
$\Delta$ PDAc-MPs & 0.667 & 0.002 & $0.553 \sim 0.781$ \\
CEA & 0.460 & 0.464 & $0.354 \sim 0.567$ \\
\hline Variables & Cut-off value & Sensitivity & Specificity \\
\hline EDAp-MPs (time 3) & 10468.5 & 0.805 & 0.638 \\
EDAc-MPs (time 3) & 3557 & 0.659 & 0.840 \\
PDAp-MPs (time 3) & 15055 & 0.610 & 0.713 \\
PDAc-MPs (time 3) & 62700.5 & 0.707 & 0.734 \\
$\Delta$ EDAp-MPs & +196 & 0.634 & 0.660 \\
$\Delta$ EDAc-MPs & +267 & 0.829 & 0.596 \\
$\Delta$ PDAp-MPs & +918.5 & 0.512 & 0.734 \\
$\Delta$ PDAc-MPs & +2448 & 0.634 & 0.670 \\
$\Delta$ CEA & +6.37 & 0.463 & 0.468 \\
\hline
\end{tabular}

NSCLC $=$ non small cell lung cancer; $\mathrm{AUC}=$ area under the curve; $\mathrm{CI}=$ confidence interval; $\mathrm{NSCLC}=$ non small cell lung cancer; PDAc-MPs = platelet-derived activated microparticles; PDAp-MPs = platelet-derived apoptotic microparticles; EDAc-MPs = endothelial derived activated microparticles; EDAp-MPs = endothelial-derived apoptotic microparticles. $\triangle$ EDAp-MPs: EDApMPs levels that three months after treatment minus initial level $\triangle$ EDAc-MPs: EDAcMPs levels that three months after treatment minus initial level $\triangle$ PDAp-MPs: PDApMPs levels that three months after treatment minus initial level $\triangle$ PDAc-MPs: PDAcMPs levels that three months after treatment minus initial level

Table 8: Predictors of progressive disease in non-small cell lung cancer patients by multivariate logistic regression analysis

\begin{tabular}{lccc}
\hline Variable & Comparison & $\mathbf{O R}^{\mathbf{b}} \mathbf{( 9 5 \% \mathbf { C I }} \mathbf{)}$ & P-value \\
\hline Body height & Per 1 unit decrease & $1.179(0.009 \sim 1.387)$ & 0.074 \\
Adrenal gland metastasis & Yes vs. No & $0.007(0.001 \sim 69.783)$ & 0.290 \\
CEA & Disease control vs. & $0.999(0.998 \sim 1.001)$ & 0.464 \\
Performance status & Progression & $0.251(0.253 \sim 1.193)$ & 0.082 \\
EGFR mutant & 2 vs. 0\&1 & $150.517(8.986 \sim 2521.118)$ & $<0.0001$ \\
EDAp-MPs (time3) & Yes vs. No & $1.000(1.000 \sim 1.000)$ & 0.412 \\
EDAc-MPs (time3) & Per 1 unit decrease & $1.001(1.000 \sim 1.002)$ & 0.022 \\
PDAp-MPs (time3) & Per 1 unit decrease & $1.000(1.000 \sim 1.000)$ & 0.327 \\
PDAc-MPs (time3) & Per 1 unit decrease & $1.000(1.000 \sim 1.000)$ & 0.887
\end{tabular}

(Continued) 


\begin{tabular}{lccc}
\hline Variable & Comparison & OR $^{\mathbf{b}} \mathbf{( 9 5 \% \mathbf { C I }} \mathbf{)}$ & P-value \\
\hline$\Delta$ EDAp-MPs & positive vs. negative & $0.557(0.052 \sim 6.004)$ & 0.630 \\
$\Delta$ EDAc-MPs & positive vs. negative & $0.285(0.033 \sim 2.468)$ & 0.254 \\
$\Delta$ PDAp-MPs & positive vs. negative & $0.074(0.006 \sim 0.892)$ & 0.040 \\
$\Delta$ PDAc-MPs & positive vs. negative & $12.32(1.130 \sim 134.32)$ & 0.039 \\
\hline
\end{tabular}

${ }^{a} \triangle E D A p-M P s:$ EDApMPs levels that three months after treatment minus initial level $\triangle$ EDAc-MPs: EDAcMPs levels that three months after treatment minus initial level $\triangle$ PDAp-MPs: PDApMPs levels that three months after treatment minus initial level $\triangle$ PDAc-MPs: PDAcMPs levels that three months after treatment minus initial level 'Odds ratio

${ }^{\mathrm{c} C o n f i d e n c e ~ i n t e r v a l ~}$

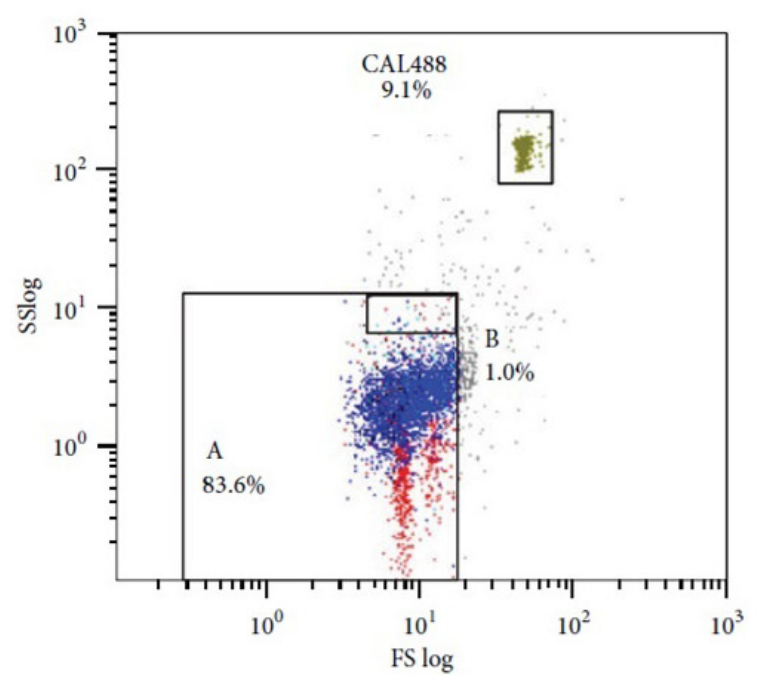

(a)



(c)

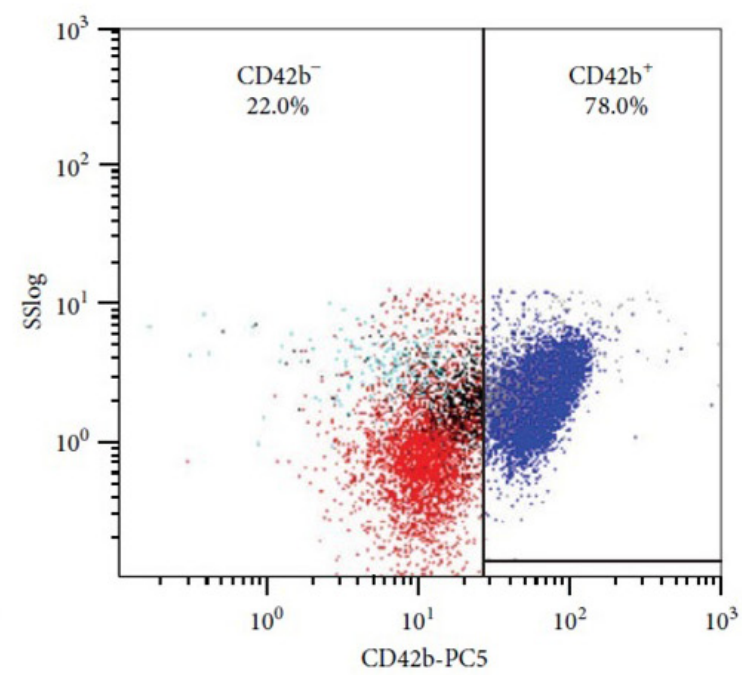

(b)

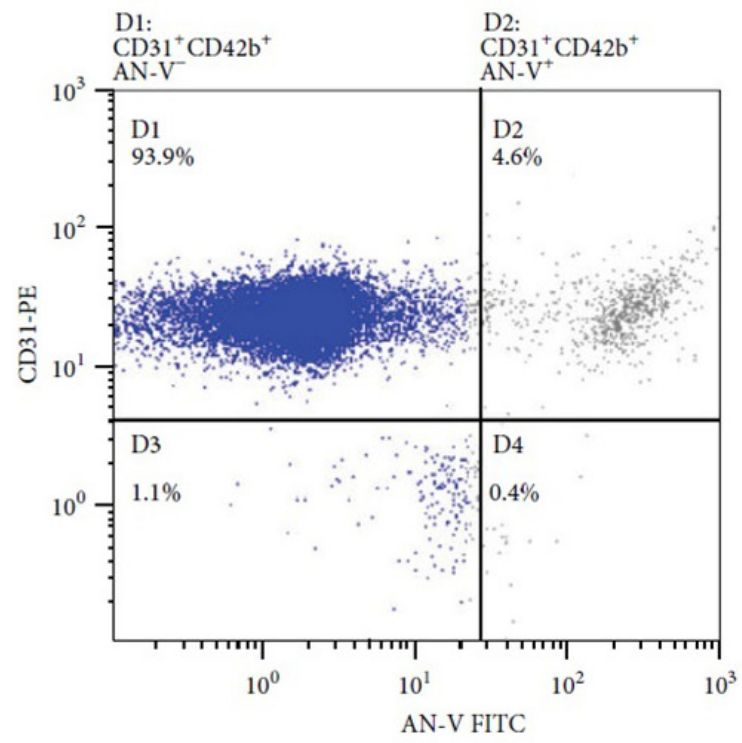

(d)

Figure 5: Flow cytometric analysis. Representative FACS plots showing the four different types of microparticles. 
associated according to previous studies [41] [18-20]. We demonstrated that the poor performance status was significantly higher in the DP group, whereas EGFR mutations were significantly lower in the DP group.

This study has limitations. First, the sample size of this cohort study was relatively small. Therefore, a new clinical trial with larger sample size is needed to validate that circulating MP levels can predict long-term clinical outcomes in advanced NSCLC patients. Second, this study measured only advanced NSCLC patients. Therefore, the usefulness of these four biomarkers in small cell lung cancer or other types of cancer patients is not known.

In conclusion, we demonstrated that serial measurement of the circulating levels of EDAc-MPs, EDAp-MPs, PDAc-MPs, PDAp-MPs predicted prognostic outcomes in advanced NSCLC patients.

\section{MATERIALS AND METHODS}

\section{NSCLC patient enrollment and therapeutic strategies}

Patient enrollment, data collection, classification of advanced stage NSCLC and therapeutic strategies were according to our previous studies [18-20]. We assessed images and pathological findings of all patients who received evaluation or treatment for $\mathrm{LC}$ at Kaohsiung Chang Gung Memorial Hospital. We determined the eligibility of patients for interventions including surgery, adjunctive or palliative chemotherapy, irradiation therapy and/or target therapy based on the AJCC cancer staging criteria, $7^{\text {th }}$ edition [39]. Based on the radiological findings, we categorized LC patients into stages I, II, IIIA, IIIB, and IV according to AJCC cancer staging criteria, $7^{\text {th }}$ edition [42]. Patients with stage IIIB or stage IV NSCLC were categorized as advanced stage NSCLC. All the patients were enrolled for further evaluation, blood sampling and treatment in the outpatient department or upon hospital admission.

Detailed in-hospital and follow-up data including age, gender, chest $\mathrm{x}$-ray findings, computed tomography, fibro-bronchoscopic findings, bone scans or ultrasound studies, other image findings, histological, pathological and laboratory findings were collected prospectively and entered into a computer database for analyses.

Informed consent was obtained from all patients and control subjects enrolled in the study. The study protocol was approved by the Institutional Review Committee on Human Research at Kaohsiung Chang Gung Memorial Hospital (IRB number: 100-1024B). The clinical investigations were conducted according to the principles outlined in the Declaration of Helsinki.

To circumvent adverse influences on measurement of circulating level of MPs, patients with one or more of the following criteria were excluded based on our previous studies [18-20]: (1) recent surgery or trauma during the preceding 2 months;(2) refusal to participate in the study; (3) other co-existent or history of malignances; (4) severe organ disease other than LC like chronic kidney disease (CKD > stage III), liver cirrhosis, hematologic disorders, congestive heart failure; (5) current use of anti-platelet agents;(6) history of febrile disorders; (7) acute or chronic inflammatory disease other than LC during the study period; or (8) a history of autoimmune diseases with or without immunosuppressive therapy.

A total of 1418 NSCLC patients were screened at Kaohsiung Chang Gung Memorial Hospital from March 2012 to January 2015. Among them, 1106 (78\%) were advanced NSCLC patients. For the purpose of the study, only patients with advanced NSCLC without prior treatment were considered. Among the 1,418 patients (including NSCLC and other type of lung cancer), 1145 patients did not fit the enrolment criteria and were excluded from the study. Additionally, 35 patients were excluded due to the aforementioned reasons. Finally, 136 patients who were diagnosed with advanced stage NSCLC between March 2012 and January 2015 were prospectively enrolled in this study [stage IIIB, $22.1 \%$ (30); stage IV, $77.9 \%$ (106)] (Table 3 ). These 136 patients were further divided into disease controlled (DC) group $(n=94)$ and disease progression (DP) group $(n=42)$.

\section{Flow cytometry analysis of circulating microparticles}

As shown in Figure 5, the circulating MPs were categorized as (1) platelet-derived activated MPs (PDAcMPs; $\left.\mathrm{CD}_{3} 1^{+} \mathrm{CD}_{2} 2 \mathrm{~b}^{+} \mathrm{AN}^{-} \mathrm{V}\right)$; (2) platelet-derived apoptotic MPs (PDAp-MPs; CD31 ${ }^{+} \mathrm{CD}_{2} 2 \mathrm{~b}^{+} \mathrm{AN}^{-} \mathrm{V}^{+}$); (3) endothelial-derived activated MPs (EDAc-MPs; CD31 ${ }^{+}$ $\mathrm{CD}^{2} \mathrm{~b}^{-} \mathrm{AN}-\mathrm{V}^{-}$); and (4) endothelial-derived apoptotic MPs (EDAp-MPs; $\mathrm{CD}^{3} 1^{+} \mathrm{CD}^{2} \mathrm{~b}^{-} \mathrm{AN}^{-} \mathrm{V}^{+}$) based on a previous study [43] with some modifications [18-20].

\section{Blood sample collection and processing}

To determine circulating levels of MPs in advanced stage NSCLC patients, blood samples were collected at 9:00 am prior to and at the end of the first and third month after therapeutic interventions according to previously published protocol [18-20]. Additionally, blood samples were also collected at 9:00 am once from control subjects.

For flow cytometry, peripheral blood was collected in acid citrate dextrose (ACD) vacutainer tubes. Plateletrich plasma was prepared by centrifuging $1.5 \mathrm{ml}$ peripheral blood at $2500 \mathrm{~g}$ at $4^{\circ} \mathrm{C}$ for $15 \mathrm{~min}$ without acceleration. Then, $250 \mu$ l plasma samples were thawed and centrifuged at $19,800 \mathrm{~g}$ for $10 \mathrm{~min}$ at $4^{\circ} \mathrm{C}$, and then collected for analyzing MPs smaller than $1.0 \mu \mathrm{m}$.

Size calibration was conducted with $1.0 \mu \mathrm{m}$ beads (Invitrogen, Carlsbad, CA). The MP pellet was re-suspended with $150 \mu 1$ of AnnexinV binding buffer 
(BD Biosciences). All buffers were sterile-filtered with a $0.2 \mu \mathrm{m}$ filter. Then, $100 \mu \mathrm{l}$ MPs were incubated in a TruCOUNT tube (BD Biosciences) with the following fluorescent monoclonal antibodies: (1) phycoerythrin (PE)-conjugated anti-CD31 (BD Biosciences); (2) fluorescein isothiocyanate-conjugated anti-AnnexinV (BD Biosciences) and; (3) phycoerythrin-Cy5 (PE-Cy5)conjugated anti-CD42b (BD Biosciences). The samples were incubated in the dark for $15 \mathrm{~min}$ at room temperature followed by addition of $400 \mu \mathrm{l}$ AnnexinV binding buffer and then analyzed in a FC500 flow cytometer (Beckman Coulter). The absolute count of MPs was measured by setting up the FACS machine with TruCount beads at 10,000 events. Additionally, white blood cell (WBC) counts, biochemistry and electrolyte levels were analyzed by standard laboratory methods in our hospital.

\section{Disease classification}

Change in tumor burden was assessed to determine tumor response to adjunctive therapy [18-20]. The chest computed tomography (CT) scans were routinely performed at baseline and at 12 week intervals after adjunctive therapy to determine the status of the disease. The tumor measurement was based on the current guidelines of Response Evaluation Criteria in Solid Tumors (RECIST) including complete response, partial response, stable disease and progressive disease [39]. Accordingly, we categorized the disease status as diseasecontrolled (DC) or disease-progressed (DP). The DC status was determined at the 3rd month after the treatment and defined as disease with regression with complete response, partial response or stable disease. On the other hand, the DP was defined as disease unresponsive to therapy with a growing tumor or metastasis after complete course of treatment. To elucidate if the changes in MPs over timecourse of treatment predicted outcomes, the relative levels (initial vs. third month) of the four types of microparticles were analyzed. The differences in the four types of microparticles were designated as $\triangle$ EDAp-MPs, $\triangle$ EDAcMPs, $\triangle$ PDAp-MPs and $\triangle$ PDAc-MPs, respectively.

\section{Statistical analysis}

Data were expressed as means \pm standard deviation (SD). Continuous variables were analyzed by independent $t$ tests and categorical variables were analyzed by the chi-square test. To determine outcomes, we compared levels of MPs three months after treatment with the initial levels. All variables were considered as risk factors with a $P<0.10$ in univariate analysis and were further analyzed by the multivariate logistic regression analysis to identify the independent factors that predict progressive disease. Receiver operating characteristic (ROC) curves were plotted and the area under the curve and CEA levels were compared for the four types of MPs. The cutoff value of MPs for predicting progressive disease in NSCLC patients was according to ROC curves. Results were presented as absolute numbers (percentage) or mean \pm SD as well as medians, interquartile ranges and ranges for the various MP results. A two-tailed $\mathrm{P}$ value of less than 0.05 was considered statistically significant. Statistical analysis was performed using SPSS statistical software for Windows version 13 (SPSS for Windows, version 13; SPSS Inc., IL).

\section{Abbreviations}

ACD: acid citrate dextrose; AJCC: American joint committee on cancer; ALK: anaplastic lymphoma kinase; $\mathrm{BD}$ : binding buffer; CAD: coronary artery disease;CEA: carcinoembryonic antigen; $\mathrm{CKD}$ : chronic kidney disease; COPD: chronic obstructive pulmonary disease; CT: computed tomography; DC: disease control; DP: disease progression; EDAc-MPs: endothelial-derived activated MPs; EDAp-MPs: endothelial-derived apoptotic MPs; EGFR: epidermal growth factor receptor; GOT: glutamic oxaloacetic transaminase; GPT: glutamic pyruvic transaminase; LC: lung cancer; MPs: microparticles; NSCLC: non-small cell lung cancer; PDAc-MPs: plateletderived activated MPs; PDAp-MPs: platelet-derived apoptotic MPs; RBC: red blood cell; RECIST: Response Evaluation Criteria in Solid Tumors; ROC: Receiver operating characteristic; SCLC: small cell lung cancer; SD: standard deviation; TKI: tyrosine kinase inhibitor; WBC: white blood cell.

\section{Author contributions}

Conception and design: Chin-Chou Wang, ChangChun Hsiao, Meng-Chih Lin, Chi-Kung Ho, and Hon-Kan Yip. Acquisition of data: Chin-Chou Wang, Chia-Cheng Tseng, Huang-Chih Chang, Kuo-Tung Huang, Wen-Feng Fang, Yu-Mu Chen, and Meng-Chih Lin. Data analysis and interpretation: Chin-Chou Wang, Chia-Cheng Tseng, Chang-Chun Hsiao, Cheng-Ta Yang, Meng-Chih Lin, Chi-Kung Ho, and Hon-Kan Yip. Manuscript writing: all authors. Final approval of manuscript: all authors.

\section{ACKNOWLEDGMENTS}

We thank Prof. Sheng-Nan Lu, Prof. Hsueh-Wen Chang, Shin-Yi Chien, Chih-Yun Lin, and the Biostatistics Center, Kaohsiung Chang Gung Memorial Hospital for statistics work.

\section{CONFLICTS OF INTEREST}

The authors declare no conflicts of interests. 


\section{FUNDING}

This study was supported by grants from the Chang Gung Memorial Hospital (CMRPG8B0011, CMRPG8B0012, CMRPG8B0013, and CMRPG8F1441 to Chia-Cheng Tseng; CMRPG8E1661, CMRPG8F1351 and CMRPG8F1491 to Chin-Chou Wang).

\section{REFERENCES}

1. Favaretto AG, Pasello G, Magro C. Second and third line treatment in advanced non-small cell lung cancer. Discov Med. 2009; 8:204-09.

2. Jemal A, Center MM, DeSantis C, Ward EM. Global patterns of cancer incidence and mortality rates and trends. Cancer Epidemiol Biomarkers Prev. 2010; 19:1893-907.

3. Marcus PM, Bergstralh EJ, Fagerstrom RM, Williams DE, Fontana R, Taylor WF, Prorok PC. Lung cancer mortality in the Mayo Lung Project: impact of extended follow-up. J Natl Cancer Inst. 2000; 92:1308-16.

4. Ramalingam SS, Owonikoko TK, Khuri FR. Lung cancer: new biological insights and recent therapeutic advances. CA Cancer J Clin. 2011; 61:91-112.

5. Maione P, Rossi A, Sacco PC, Bareschino MA, Schettino $\mathrm{C}$, Gridelli C. Advances in chemotherapy in advanced nonsmall-cell lung cancer. Expert Opin Pharmacother. 2010; 11:2997-3007.

6. Maemondo M, Inoue A, Kobayashi K, Sugawara S, Oizumi $\mathrm{S}$, Isobe $\mathrm{H}$, Gemma A, Harada M, Yoshizawa H, Kinoshita I, Fujita Y, Okinaga S, Hirano H, et al, and North-East Japan Study Group. Gefitinib or chemotherapy for non-small-cell lung cancer with mutated EGFR. N Engl J Med. 2010; $362: 2380-88$.

7. Mok TS, Wu YL, Thongprasert S, Yang CH, Chu DT, Saijo N, Sunpaweravong P, Han B, Margono B, Ichinose Y, Nishiwaki Y, Ohe Y, Yang JJ, et al. Gefitinib or carboplatinpaclitaxel in pulmonary adenocarcinoma. N Engl J Med. 2009; 361:947-57.

8. Rosell R, Carcereny E, Gervais R, Vergnenegre A, Massuti B, Felip E, Palmero R, Garcia-Gomez R, Pallares C, Sanchez JM, Porta R, Cobo M, Garrido P, et al, and Spanish Lung Cancer Group in collaboration with Groupe Français de Pneumo-Cancérologie and Associazione Italiana Oncologia Toracica. Erlotinib versus standard chemotherapy as first-line treatment for European patients with advanced EGFR mutation-positive non-small-cell lung cancer (EURTAC): a multicentre, open-label, randomised phase 3 trial. Lancet Oncol. 2012; 13:239-46.

9. Zhou C, Wu YL, Chen G, Feng J, Liu XQ, Wang C, Zhang S, Wang J, Zhou S, Ren S, Lu S, Zhang L, Hu C, et al. Erlotinib versus chemotherapy as first-line treatment for patients with advanced EGFR mutation-positive non-smallcell lung cancer (OPTIMAL, CTONG-0802): a multicentre, open-label, randomised, phase 3 study. Lancet Oncol. 2011; $12: 735-42$.
10. Kwak EL, Bang YJ, Camidge DR, Shaw AT, Solomon B, Maki RG, Ou SH, Dezube BJ, Jänne PA, Costa DB, VarellaGarcia M, Kim WH, Lynch TJ, et al. Anaplastic lymphoma kinase inhibition in non-small-cell lung cancer. N Engl J Med. 2010; 363:1693-703.

11. Arriagada R, Bergman B, Dunant A, Le Chevalier T, Pignon JP, Vansteenkiste J, and International Adjuvant Lung Cancer Trial Collaborative Group. Cisplatin-based adjuvant chemotherapy in patients with completely resected non-small-cell lung cancer. N Engl J Med. 2004; 350:351-60.

12. Spiro SG, Silvestri GA. One hundred years of lung cancer. Am J Respir Crit Care Med. 2005; 172:523-29.

13. Stinchcombe TE, Socinski MA. Maintenance therapy in advanced non-small cell lung cancer: current status and future implications. J Thorac Oncol. 2011; 6:174-82.

14. Veeramachaneni NK, Feins RH, Stephenson BJ, Edwards LJ, Fernandez FG. Management of stage IIIA non-small cell lung cancer by thoracic surgeons in North America. Ann Thorac Surg. 2012; 94:922-26.

15. Chanin TD, Merrick DT, Franklin WA, Hirsch FR. Recent developments in biomarkers for the early detection of lung cancer: perspectives based on publications 2003 to present. Curr Opin Pulm Med. 2004; 10:242-47.

16. Hassanein M, Callison JC, Callaway-Lane C, Aldrich MC, Grogan EL, Massion PP. The state of molecular biomarkers for the early detection of lung cancer. Cancer Prev Res (Phila). 2012; 5:992-1006.

17. Herbst RS, Onn A, Sandler A. Angiogenesis and lung cancer: prognostic and therapeutic implications. J Clin Oncol. 2005; 23:3243-56.

18. Tseng CC, Wang CC, Chang HC, Tsai TH, Chang LT, Huang KT, Leu S, Yen CH, Liu SF, Chen CH, Yang CT, Yip HK, Lin MC. Levels of circulating microparticles in lung cancer patients and possible prognostic value. Dis Markers. 2013; 35:301-10.

19. Tseng CC, Wang CC, Hsiao CC, Lu HI, Leu S, Chang HC, Huang KT, Fang WF, Chen YM, Liu SF, Yang CT, Lin MC, Yip HK. Time courses and value of circulating microparticles in patients with operable stage non-small cell lung cancer undergoing surgical intervention. Tumour Biol. 2016; 37:11873-82.

20. Wang CC, Tseng CC, Hsiao CC, Chang HC, Chang LT, Fang WF, Leu S, Wang YH, Tsai TH, Yang CT, Chen CH, Yip HK, Ho CK, et al. Circulating endothelial-derived activated microparticle: a useful biomarker for predicting one-year mortality in patients with advanced non-small cell lung cancer. Biomed Res Int. 2014; 2014:173401. https:// doi.org/10.1155/2014/173401.

21. Fleitas T, Martínez-Sales V, Vila V, Reganon E, Mesado D, Martín M, Gómez-Codina J, Montalar J, Reynés G. Circulating endothelial cells and microparticles as prognostic markers in advanced non-small cell lung cancer. PLoS One. 2012; $7:$ e47365. 
22. Horstman LL, Ahn YS. Platelet microparticles: a wide-angle perspective. Crit Rev Oncol Hematol. 1999; 30:111-42.

23. Mallat Z, Benamer H, Hugel B, Benessiano J, Steg PG, Freyssinet JM, Tedgui A. Elevated levels of shed membrane microparticles with procoagulant potential in the peripheral circulating blood of patients with acute coronary syndromes. Circulation. 2000; 101:841-43.

24. Martin S, Tesse A, Hugel B, Martínez MC, Morel O, Freyssinet JM, Andriantsitohaina R. Shed membrane particles from $\mathrm{T}$ lymphocytes impair endothelial function and regulate endothelial protein expression. Circulation. 2004; 109:1653-59.

25. Martínez MC, Larbret F, Zobairi F, Coulombe J, Debili N, Vainchenker W, Ruat M, Freyssinet JM. Transfer of differentiation signal by membrane microvesicles harboring hedgehog morphogens. Blood. 2006; 108:3012-20.

26. Martínez MC, Tesse A, Zobairi F, Andriantsitohaina R. Shed membrane microparticles from circulating and vascular cells in regulating vascular function. Am J Physiol Heart Circ Physiol. 2005; 288:H1004-09.

27. Mezentsev A, Merks RM, O'Riordan E, Chen J, Mendelev N, Goligorsky MS, Brodsky SV. Endothelial microparticles affect angiogenesis in vitro: role of oxidative stress. Am J Physiol Heart Circ Physiol. 2005; 289:H1106-14.

28. Mostefai HA, Andriantsitohaina R, Martínez MC. Plasma membrane microparticles in angiogenesis: role in ischemic diseases and in cancer. Physiol Res. 2008; 57:311-20.

29. Nomura S. Function and clinical significance of plateletderived microparticles. Int J Hematol. 2001; 74:397-404.

30. Nomura S, Ozaki Y, Ikeda Y. Function and role of microparticles in various clinical settings. Thromb Res. 2008; 123:8-23.

31. Zwaal RF, Schroit AJ. Pathophysiologic implications of membrane phospholipid asymmetry in blood cells. Blood. 1997; 89:1121-32.

32. Nomura S, Uehata S, Saito S, Osumi K, Ozeki Y, Kimura Y. Enzyme immunoassay detection of platelet-derived microparticles and RANTES in acute coronary syndrome. Thromb Haemost. 2003; 89:506-12.

33. Daniel L, Fakhouri F, Joly D, Mouthon L, Nusbaum P, Grunfeld JP, Schifferli J, Guillevin L, Lesavre P, Halbwachs-Mecarelli L. Increase of circulating neutrophil and platelet microparticles during acute vasculitis and hemodialysis. Kidney Int. 2006; 69:1416-23.
34. Janowska-Wieczorek A, Marquez-Curtis LA, Wysoczynski M, Ratajczak MZ. Enhancing effect of platelet-derived microvesicles on the invasive potential of breast cancer cells. Transfusion. 2006; 46:1199-209.

35. Joop K, Berckmans RJ, Nieuwland R, Berkhout J, Romijn FP, Hack CE, Sturk A. Microparticles from patients with multiple organ dysfunction syndrome and sepsis support coagulation through multiple mechanisms. Thromb Haemost. 2001; 85:810-20.

36. Nomura S, Kagawa H, Ozaki Y, Nagahama M, Yoshimura C, Fukuhara S. Relationship between platelet activation and cytokines in systemic inflammatory response syndrome patients with hematological malignancies. Thromb Res. 1999; 95:205-13.

37. Ogata N, Imaizumi M, Nomura S, Shozu A, Arichi M, Matsuoka M, Matsumura M. Increased levels of plateletderived microparticles in patients with diabetic retinopathy. Diabetes Res Clin Pract. 2005; 68:193-201.

38. Tesselaar ME, Romijn FP, Van Der Linden IK, Prins FA, Bertina RM, Osanto S. Microparticle-associated tissue factor activity: a link between cancer and thrombosis? J Thromb Haemost. 2007; 5:520-27.

39. Therasse P, Arbuck SG, Eisenhauer EA, Wanders J, Kaplan RS, Rubinstein L, Verweij J, Van Glabbeke M, van Oosterom AT, Christian MC, Gwyther SG. New guidelines to evaluate the response to treatment in solid tumors. European Organization for Research and Treatment of Cancer, National Cancer Institute of the United States, National Cancer Institute of Canada. J Natl Cancer Inst. 2000; 92:205-16.

40. Herbst RS, Heymach JV, Lippman SM. Lung cancer. N Engl J Med. 2008; 359:1367-80.

41. Simmons CP, Koinis F, Fallon MT, Fearon KC, Bowden J, Solheim TS, Gronberg BH, McMillan DC, Gioulbasanis I, Laird BJ. Prognosis in advanced lung cancer-A prospective study examining key clinicopathological factors. Lung Cancer. 2015; 88:304-09.

42. Edge SB, Compton CC. The American Joint Committee on Cancer: the 7th edition of the AJCC cancer staging manual and the future of TNM. Ann Surg Oncol. 2010; 17:1471-4. https://doi.org/10.1245/s10434-010-0985-4.

43. Dey-Hazra E, Hertel B, Kirsch T, Woywodt A, Lovric S, Haller H, Haubitz M, Erdbruegger U. Detection of circulating microparticles by flow cytometry: influence of centrifugation, filtration of buffer, and freezing. Vasc Health Risk Manag. 2010; 6:1125-33. 